\title{
Fan Engagement in 15 Seconds: Athletes' Relationship Marketing During a Pandemic via TikTok
}

\author{
Yiran Su \\ University of Georgia \\ Jason P. Doyle
Griffith University
}

\author{
Bradley J. Baker \\ University of Massachusetts Amherst
}

\author{
Meimei Yan \\ University of Georgia
}

\begin{abstract}
As COVID-19 lockdowns force most sport leagues into hiatus, engaging fans has emerged as a key challenge confronting the sport industry. While navigating social distancing protocols, athletes are experimenting with new ways to connect with their fans. Alongside established social media platforms (e.g., Twitter, Facebook, and Instagram), TikTok, a short-form video-sharing platform, has gained prominence in terms of registered users and shared content. Yet, little is known about the utility of TikTok as an athlete branding tool. This study uses a netnographic approach to explore the use of TikTok among athletes $(N=10)$ during the COVID-19 pandemic. Findings reveal that athlete-generated TikTok videos are characterized as playful and authentic. While athletes are recent adopters of TikTok, this emerging social media platform can be profitably integrated into their online branding strategies. Communicating via TikTok presents opportunities for athletes to foster existing fan relationships, promote branded content, and appeal to new fan segments. Overall, athletes and sport practitioners can leverage these findings to create content for an audience that is attracted to novelty and the activities of athletes extending beyond game highlights or interviews.
\end{abstract}

Keywords: athlete branding, COVID-19, social media, sport consumers

With nearly all sport across the world paused due to the coronavirus, athletes have demonstrated their creativity during quarantine by experimenting with new training routines, at-home entertainment options, and new social media platforms to connect with fans. The absence of original sport-related content due to

\footnotetext{
Su and Yan are with the Dept. of Kinesiology, University of Georgia, Athens, GA, USA. Baker is with the Mark H. McCormack Dept. of Sport Management, University of Massachusetts Amherst, Amherst, MA, USA. Doyle is with the Dept. of Tourism, Sport, and Hotel Management, Griffith University, Nathan, QLD, Australia. Su (yiransu@uga.edu) is corresponding author.
} 
constraints on social gatherings has posed challenges for athletes to maintain fan relationships. Against this background, TikTok has gained prominence as the latest digital apparatus for fan engagement. Athlete-generated TikTok videos have been widely reported on by news outlets. Examples include Lebron James' choreographed dances with his family and numerous Women's National Basketball Association players joining the \#DontRushChallenge (Women's National Basketball Association, 2020). While scholars have assessed athletes' use of established social media platforms such as Twitter, Facebook, Instagram, and, more recently, Snapchat, little is known about the utility of TikTok as a strategic communication tool. In light of the increasing popularity TikTok has gained amid COVID-19, the present commentary explores athletes' use of TikTok as a communication platform during this pandemic with the intention of reflecting the changing nature of sport communication and branding in this challenging time.

Although not widely adopted in sport, TikTok had already demonstrated its utility as a social media platform prepandemic. Together with its Chinese version, Douyin, this short video-sharing app has been downloaded more than 2 billion times globally (Leskin, 2020). Such popularity has caught the attention of sport marketers, tapping into the app's Generation $\mathrm{Z}$ and female user bases in particular (Moran, 2019). Prior to the lockdown, the major American sport leagues started cultivating their presence on TikTok. Notable examples include hashtag challenges using \#TouchdownCelebration during the run-up to the 2020 Super Bowl and performances by TikTok influencers at the National Basketball Association All-Star Game. Moreover, while TikTok is still testing in-app e-commerce functions in the United States, Douyin has proven a powerful marketing medium in China with sport entities and athletes selling sponsored products and promoting branded content.

As the lockdown supercharges adoption of the TikTok app among athletes, TikTok warrants examination as a new way of communicating with fans. Despite theoretical appeal and practitioner relevance, investigations into the use of TikTok in sport are only beginning to emerge in the literature. In this scholarly commentary, we adopt a netnographic approach to explore the potential of TikTok as an emerging fan engagement and self-branding tool. In doing so, we ask the following three questions: (a) How are athletes using TikTok to engage fans during the pandemic? (b) What elements make TikTok a distinct engagement tool for athletes? and (c) What is the role of TikTok in athletes' branding efforts? Our results shed light on social media management during the coronavirus pandemic and enhance understanding of the mechanisms by which TikTok may effectively engage sport fans.

\section{Background and Theoretical Foundation}

\section{TikTok and COVID-19}

TikTok is a social media app focusing on short video sharing. Users can take advantage of an assortment of templates, filters, and visual effects, as well as a builtin music library, to create short videos. The most popular video genres are lipsyncing, dancing, or acting out comedic skits set to 15-second music clips. Following the success of its Chinese sister app Douyin, TikTok is gradually moving toward a more commercialized social media platform, allowing users to earn revenue from in-app purchases and paid reactions or gifts in response to their videos. 
With most people staying at home, TikTok has enjoyed phenomenal growth amid the COVID-19 pandemic. The app added 12 million U.S. users in March 2020 and a total of 52.2 million users globally (Weiss, 2020), making it the most downloaded nongaming app on the Apple app store in Q1, 2020 (Leskin, 2020). Time spent on the app and its websites has also surged. For example, U.S. visitors spent an average of 8 hours on the app during the month of March, which is $10.8 \%$ higher than time spent in January (Weiss, 2020). Alongside this surge in popularity, athletes started exploring TikTok as a means to engage with fans and ensure their brands are receiving attention during this pause in competition. The humorous and casual nature of TikTok videos represents a source of comfort and a time killer while self-isolating and social distancing during the pandemic. Moreover, TikTok represents a new way for athletes to build their brands.

\section{Athlete Branding and Social Media}

Human brand theory (Thomson, 2006) suggests that consumers form strong attachments to well-known personas that are attractive, accessible, and authentic, yielding positive marketing results. Athletes' personal brands are among their most valuable assets (Su, Baker, Doyle, \& Kunkel, 2020) and positive brand associations continue even after athletic performance declines (Pegoraro \& Jinnah, 2012) or ceases, as evidenced by the persistent marketability of retired athletes, such as Michael Jordan, David Beckham, and Mia Hamm. Thus, athletes are presented with incentives to establish a strong brand presence in the mind of consumers. One ubiquitous context for personal brand building is social media.

The importance of social media in sport business manifests in how it supports engagement between athletes and fans. Social media has transformed the relationship between athletes and fans, reducing perceived barriers and bringing the two closer together (Pegoraro, 2010). Athletes use social media to share their personal lives, develop connections with fans, gain sponsorships, and promote themselves (Geurin, 2017). Moreover, social media not only serves as a platform for athletes to present an image of themselves, but also allows fans to interact, comment, and otherwise engage with athletes (Geurin-Eagleman \& Burch, 2016). Regarding the effectiveness of social media posts, consumers appreciate genuineness and are "interested in the real, unmanicured persona of the athlete" (Frederick \& Clavio, 2015, p. 340), rather than more curated content (cf. Ilicic \& Webster, 2016). Therefore, strategic management of social media accounts represents a key component in athletes' branding arsenal (Geurin, 2017).

\section{Method}

To answer our research questions, we took a netnographic and comparative approach (Kozinets, 2010) to analyze the TikTok phenomenon with a focus on athlete branding. A netnographic approach allowed us to unobtrusively collect data and uncover patterns of behavior that are situated in a broader cultural and social context (Kozinets, 2010). As Douyin predates TikTok and has a longer track record, this comparison helped us better benchmark the way TikTok is currently used by U.S.-based athletes and explore its future potential. 
We began by taking a broad approach to observing how professional athletes are using TikTok and Douyin and the associated responses this content attracts from users, including comments, likes, reposts, and views. We additionally reviewed mainstream media reports discussing athletes' use of each platform. This provided us a sense of which professional athletes have the highest profile TikTok accounts. Based on this initial review, we selected 10 athletes-five U.S.based and five China-based - as a microlevel case study (see Table 1 for the full list of athletes). These athletes were purposefully selected to reflect both the United States and Chinese sport contexts and included male and female athletes across a range of individual and team-based sports. In selecting athletes, we sought diversity in terms of gender, sport, and background, while ensuring that each athlete was well-established within their sport, actively used social media for professional self-branding, and attracted a sizeable TikTok audience.

We downloaded all postings during the shelter-in-place orders. That is, between March 14 and May 9, 2020 for TikTok $(n=67)$ and between January 24 and May 9, 2020 for Douyin ( $n=99$; a nationwide shutdown started earlier in China). Following previous research on athletes' social media behavior (e.g., Geurin-Eagleman \& Burch, 2016), we performed a content analysis on these posts, coding their quantitative indicators (e.g., likes and views) and post content (e.g., sport-related or family-oriented posts). ${ }^{1}$ Beyond the coding process, all authors reviewed the videos, browsed viewers' comments, and provided field notes, aligned with the nature of netnographic inquiry. We further expanded our

\section{Table 1 General Information on the Selected Athletes From TikTok and Douyin}

\begin{tabular}{|c|c|c|c|c|c|}
\hline Platform & Athlete & Handle & Gender & Sport & Followers \\
\hline \multirow[t]{5}{*}{ TikTok } & $\begin{array}{l}\text { Giannis } \\
\text { Antetokounmo }\end{array}$ & @ giannis_an34 & Male & Basketball & 960,100 \\
\hline & Alex Bregman & @abreg_1 & Male & Baseball & 352,300 \\
\hline & $\begin{array}{l}\text { Julie Ertz and } \\
\text { Zach Ertz }\end{array}$ & @ teamertz ${ }^{\mathrm{a}}$ & $\begin{array}{l}\text { Female } \\
\text { and male }\end{array}$ & $\begin{array}{l}\text { Soccer and } \\
\text { football }\end{array}$ & 62,600 \\
\hline & $\begin{array}{l}\text { Laurie } \\
\text { Hernandez }\end{array}$ & @ lauriehernandez & Female & Gymnastics & 514,200 \\
\hline & LeBron James & @bryce23james ${ }^{\text {b }}$ & Male & Basketball & 549,900 \\
\hline \multirow[t]{5}{*}{ Douyin } & Ruoqi Hui & @huigelivable & Female & Volleyball & 478,000 \\
\hline & Jeremy Lin & @625721948 & Male & Basketball & $6,907,000$ \\
\hline & $\begin{array}{l}\text { Stephen } \\
\text { Marbury }\end{array}$ & @ Starbury & Male & Basketball & $1,402,000$ \\
\hline & Minxia Wu & @wuminxia1110 & Female & Diving & 763,000 \\
\hline & Jike Zhang & @Zhangjike999 & Male & $\begin{array}{l}\text { Table } \\
\text { tennis }\end{array}$ & $2,627,000$ \\
\hline
\end{tabular}

Note. Number of followers as of May 9, 2020.

a Julie Ertz and Zach Ertz jointly manage their TikTok account named Team Ertz. ${ }^{b}$ LeBron and Savannah James manage the TikTok account of their son Bryce; the James family collaborate on content. 
scope of analysis by comparing the athletes' behavior on other major social media outlets to their TikTok usage.

\section{Findings}

The sequential, incremental netnographic exploration provided insight into how athletes are using TikTok during the quarantine and the role of TikTok in athletes' self-branding efforts. We report findings related to our research questions in the following sections.

\section{How Are Athletes Using TikTok to Engage Fans During the Pandemic?}

The U.S.-based athletes we examined started to use or became newly active again on TikTok during the lockdown. For example, prior to the COVID-19 shelter-inplace orders, the last time Laurie Hernandez had posted was January 5, 2020; she resumed active posting to TikTok with four videos posted on March 20, 2020. Content-wise, most of the posts on TikTok were not related to sport, but more reflective of the personal side of the athlete. The U.S.-based athletes took advantage of many of TikTok's unique features within their posts, leveraging the visual effects within choreographed dances or simply while portraying their daily life at home during the lockdown. For instance, a video posted by Julie and Zach Ertz showed a funny conversation between Zach, who was washing the dishes, and Julie, who was lying on the couch. The athlete couple lip-synced audio from Keeping up with the Kardashians, ${ }^{2}$ turning routine housework into an interesting and humorous snapshot for TikTok users.

In addition to personal life posts, the athletes also posted performance-related content, offering a glimpse into their new at-home training routines. This type of content echoes well with the challenge function on TikTok, which is a taskoriented hashtag associated with filming a video with a specific set of movements to the same song. Athletic challenges are popular on TikTok, providing athletes the opportunity to showcase their abilities and attract attention from other users. One such video that went viral on TikTok is the "clock challenge" posted by gymnast Laurie Hernandez. The handstand video features the numbers on a clockface marking where Laurie moves next using her legs to create a wide circle, matching the beat of Kanye West's popular song Stronger.

Finally, the athletes posted game highlight videos from before the shutdown, expressing their longing for competition and the return of sport. While not a consistent focus of their content, some athletes mentioned "quarantine" or "pandemic" in their posts, including videos and captions. For instance, Alex Bregman posted his quarantine workout routine. This indicates that athletes use their posts to join in contemporary conversations and exert positivity, reflecting the potential for TikTok as a trendy social media platform amidst the pandemic.

Interestingly, all U.S.-based athletes we reviewed recorded videos in collaboration with family members or teammates. For example, the James family dancing together received extensive media exposure and positive feedback. Similarly, Julie and Zach Ertz are both famous athletes in their respective fields (soccer and 
football) and created a joint account to document their daily life on TikTok during the quarantine. The theme of family and friends, highlighting human connection, is in the spotlight during the pandemic. This could explain why athletes choose this type of content and the rising popularity of the videos. From a branding perspective, these videos also exemplify co-branding between individual personal brands. Athletes use TikTok videos to showcase a behind the scenes and relatable look at their personal lives and to demonstrate a side of their brand that complements their professional images.

Compared with the U.S.-based athletes' posting patterns on TikTok, Chinabased athletes displayed a more sophisticated usage of Douyin, exemplified by more regular and frequent posting, a complex application of visual effects, and the promotion of sponsored products or events. Many China-based athletes have developed their own commercialized workout programs, including a series of videos posted on Douyin. For instance, Minxia Wu was invited by Douyin to teach online fitness courses during the quarantine. This shows that Chinabased athletes have readily used Douyin as a promotional tool, indicating that Douyin is more advanced in providing commercialization opportunities than TikTok.

It is noteworthy that among our sample of China-based athletes, three have announced retirement in the past 2 years. Although this is a limitation of our data collection, it also sheds light on Douyin being an effective medium to engage fans for postcareer athletes, as these athletes continue to be widely followed and discussed on the platform. Additionally, China-based athletes have limited options on social media (i.e., Weibo and Douyin) to promote themselves, whereas U.S.based athletes have more platforms for self-branding, including Twitter, Facebook, SnapChat, YouTube, and Instagram. This disparity also contributes to Chinese athletes demonstrating a more deliberate use of short-form videos with the intention of exploiting opportunities presented by Douyin.

\section{What Elements Make TikTok a Distinct Engagement Tool for Athletes?}

Overall, our observations reveal that while many athletes are new to TikTok, some notable features of TikTok have distinguished it from more established social media platforms. Our netnographic analysis identifies two themes underpinning the key elements that distinguish TikTok. These themes, Playfulness and Performativity and Authenticity, are now discussed.

Playfulness and Performativity. Athlete TikTok videos are predominantly characterized as playful and entertaining. Unlike its biggest rival, Instagram, TikTok is more of an entertainment platform with short-form performative videos, rather than a lifestyle platform where the user chronicles their life events mainly through statements and photos. Another unique facet of TikTok is its configurable or copycat culture-it is common to reappropriate an existing clip by mixing additional visual effects or layering atop in a newly recorded video. The content is less cultivated but more spontaneous and funnier, thereby aligning with factors that contribute to virality on the Internet (Khan \& Vong, 2014). For example, a TikTok video showing Alex Bregman's fiancée pulling a prank on him was widely 
reposted across different social media platforms and reported by traditional media outlets. The emphasis on playfulness attracts a younger audience, with two thirds of TikTok users being under 30 years of age (Moran, 2019). We found that TikTok users perceive these videos as funny and some fans strive for interaction, asking athletes to respond to their comments.

Authenticity. On TikTok, self-image is presented in a less-polished way, mostly by amateur performers, compared with other social media platforms rife with professional production quality and preening influencers. The video-first platform leaves little room for users to retouch their look and the raw imagery on TikTok makes it more relatable and genuine. Particularly during the pandemic, most of the posts were filmed at home, restricting athletes' ability to portray a glamorous and exciting lifestyle. The posts we examined included athletes washing dishes, cradling infants, and playing the ukulele, demonstrating TikTok provides athletes an alternative platform to express themselves during abnormal times.

Interestingly, TikTok is not praised for originality owing to the norms of content appropriation. Yet, this platform is a place where users will find authentic self-presentation under the guise of comedic videos. Furthermore, contrary to Instagram, which emphasizes perfection within posts, TikTok users applaud selfdeprecating revelations. A notable example is LeBron James pointing out his awkwardness in choreographed TikTok dances. When reposting this TikTok video on his Twitter account, he used the caption: "TikTok/Kids + me $=$ me looking like a damn fool! [Rolling on the floor laughing] [Facepalming] [Shrugging]." 3

This self-deprecating humor, coupled with the funny dance video, closes the distance between fans and the athlete and adds a level of authenticity to the athlete's brand. In this case, it shows a personal and light-hearted side to an athlete who is usually associated with perfection and dominance on the court. Users indicated that this down-to-earth content made them feel like LeBron James was highly relatable to their own experience and lives, posting comments such as "I'm this family" [jordynkelis ${ }^{4}$ ]. In contrast to athletes' high-level professionalism on the field, their imperfect performance in TikTok videos is perceived by the audience as authentic self-expression, which elevates fan engagement (cf. Ilicic \& Webster, 2016).

\section{What Is the Role of TikTok in Athletes' Branding Efforts?}

Despite the fact that TikTok has garnered significant attention and interest during the COVID-19 quarantine, our findings indicate that U.S.-based athletes are still at an early stage of adopting this emerging branding tool. To date, TikTok has not been fully integrated into athlete self-branding portfolios, nor perceived as a major fan engagement channel. This is evidenced by the posting frequency across different platforms. While athletes are posting more content during shelter-in-place, their posts on TikTok are less frequent and regular than on other platforms.

TikTok additionally fills a role as a content generator. Thanks to numerous easy-to-use templates and unique visual effects, athletes record short TikTok 
videos and then cross-post on Instagram and Twitter, engaging their fans via established platforms. Our findings show that views of TikTok videos on Instagram were higher than on TikTok, the original platform. This suggests that TikTok can be profitably used as a social media tool to foster fan engagement and brand development while also strategically improving the performance of other social media platforms. Fans also left comments on TikTok such as, "I am here because of your Instagram/Twitter posts." Further, in contrast to what we observed on Douyin, little sponsorship-related content was posted by athletes on TikTok. This is in part because brands and celebrities are just starting to translate the user engagement to sales. In addition, TikTok has not officially launched its in-app ecommerce function as Douyin has done in China, outlining the future potential for TikTok to be integrated into an athlete's online branding suite.

\section{Discussion}

The purpose of this scholarly commentary was to explore how athletes have adopted TikTok and used it to engage fans during the COVID-19 self-quarantine period. In doing so, we discuss the reasons behind TikTok's increasing popularity during COVID-19 and the potential of TikTok as a self-branding outlet for athletes. To the best of our knowledge, this is the first time TikTok has been investigated in sport management research. Notably, many athletes were new to TikTok due to COVID-19; therefore, the preliminary findings presented in this paper serve as an essential step toward a better understanding of how sport practitioners could develop a TikTok marketing strategy.

Over the past decade, self-branding practices have become endemic to professional athletes, facilitated by the ubiquitous use of social media (Geurin, 2017). Through this scholarly commentary, we demonstrate how athletes may fill the void when traditional sport content is not available by creating personalized content that they can share via social media platforms. This content is largely nonsport-related, providing athletes with the opportunity to showcase a different side of their lives, and fans an opportunity to consume and engage with content dissimilar to what is typically posted on other platforms and in normal societal settings (i.e., nonpandemic). From a strategic branding perspective, we believe that athlete use of TikTok can be enhanced and should continue to provide followers with ongoing insights into their personalities and interests after COVID-19 restrictions are lifted. By developing a strategy leveraging the strengths of various social media platforms and by posting diverse content types, we believe athletes will be best positioned to develop strong brands with broad appeal and futureproof themselves against any future scenarios where they may not be able to provide sport-related content (e.g., in the event of injury or during other sport hiatus periods).

Our findings reveal a new approach to fan engagement via social media. TikTok's raison d'être is creating easy, engaging content for pure entertainment, manifested in the company's mottos "Everyone is a creator" and "Real People. Real Videos." From a branding perspective, TikTok's popularity attests to a shift in consumer culture where authentic brand narratives are valued and self-brand connections are more important than ever (Kunkel, Biscaia, Arai, \& Agyemang, 
2019). This could explain why Lebron James's inexperienced dance moves became viral content on, and beyond, TikTok. In such cases, professional athletes' self-deprecating, humorous presentation echoes with TikTok users seeking genuineness and authenticity.

Therefore, the key to fan engagement on TikTok is not to repost what has been presented on other social media or personal websites, where filtered and curated life updates and career achievements dominate (cf. Frederick \& Clavio, 2015). Instead, TikTok is a new way to present creative off-the-field content that can be further promoted across established platforms. While a direct comparison of TikTok and other social media platforms was not a key focus of this work, exploring athletes' digital brand profiles provided unique insights. Arguably, Facebook, Twitter, and Instagram each have their own distinct focus-Facebook and Twitter are primarily text based, and Instagram is mostly photo based. Our findings suggest that what TikTok adds to social media branding is not merely being a new video-centric platform. Our observations suggest that the distinct characteristics of TikTok, such as easy-to-apply memes and the algorithm that promotes grassroots content, are uniquely suited to the creation of viral content, which moves this video-sharing app beyond a bite-size version of YouTube or a permanent version of Instagram stories. TikTok's innovativeness was reflected in the confluence of themes presented in the "Findings" section and its relationship with other popular social media platforms, as shown in Figure 1.

Our findings further advance understanding of fan engagement in the everevolving digital era and the complexity of human brand authenticity. We argue that from an athlete branding point of view, Instagram, Facebook, and Twitter may be best used to project the professional side of an athlete's brand, while TikTok reflects a more casual, laid-back facet of the athlete's brand. While all social media platforms involve impression management, TikTok presents less staged content. As younger consumers gravitate toward genuineness, TikTok will be instrumental in fostering an engaged fan relationship, tapping into new audiences, and promoting branded content.

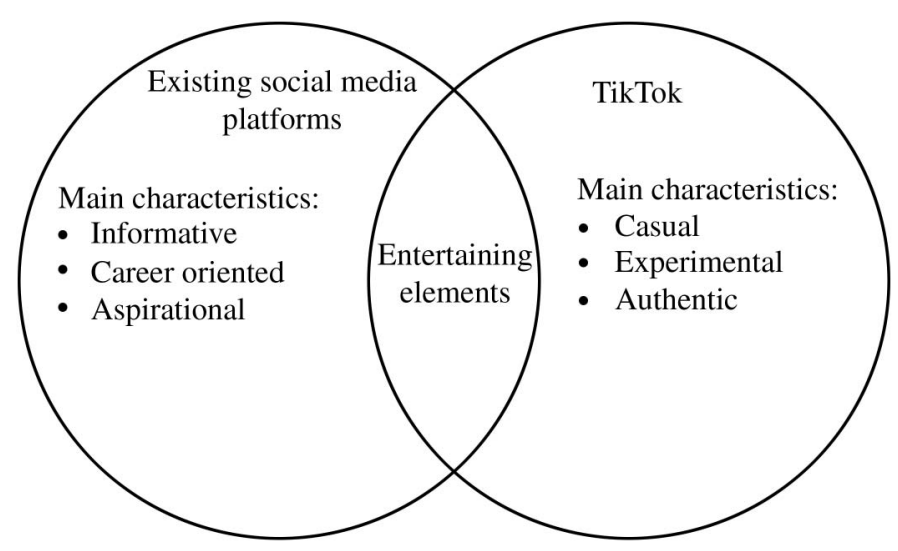

Figure 1 - Relationship between TikTok and existing social media platforms. 


\section{Future Directions}

As the outbreak of COVID-19 has brought epochal changes, the sport world is confronting uncertainty due to event cancellation, postponement, and a need for novel ways of engaging fans. This study offers additional directions for future research. The first is to investigate the role of TikTok in engaging viewers remotely and virtually when attending live sporting events is not feasible. Major professional sport leagues are attempting to gradually resume their disrupted schedules without fans in attendance. It would be interesting to examine how TikTok, known for sensory richness, could help with involving fans in competitions within empty stadiums. This is particularly relevant as TikTok is adding augmented reality functions for enhanced interactive visual effects. Will TikTok deliver "authentic" game day experiences? Will athletes post game-day content in a different way on TikTok? Will athletes continue to use TikTok for backstage or (nominally) private life content? Is the current focus driven by lack of access to sport content and greater time with family, or a persistent aspect of how athletes will continue to use the platform as the sport world resumes normal operations? These questions should all be addressed in future scholarship.

Most of the athlete-generated content we reviewed was native to TikTok. That is, the videos were originally recorded via the TikTok app, using the built-in features and tools. Many of these videos were subsequently reposted across other social media platforms (e.g., Twitter, Instagram, Facebook, and YouTube). As each has distinct roles and uses, future research should examine how TikTok fits within this ecosystem. A related research area could be investigating synergies between different platforms and how athletes can use a repertoire of social media platforms to optimize their personal brands.

Another direction is to explore the interaction between league, team, and athlete branding behavior on TikTok. As well as impacting athletes, COVID-19 has led an increasing number of sport entities to jump on the TikTok bandwagon. Recent examples include Bayern Munich announcing an official partnership with Douyin to better engage the club's satellite fans in China. This collaboration evidences the potential of the short-form video platform. The interaction on TikTok between different sport brands within the sport brand ecosystem (e.g., Kunkel \& Biscaia, 2020) would be a timely and important research topic to help sport marketers deploy efficient social media strategies with shrinking resources. As TikTok begins to allow shoppable video posts, teams and athletes can create a new source of revenue in the era of social distancing. With many athletes and sport entities new to TikTok and experimenting with ways to leverage this emerging social media platform, it opens up a channel for sport media and marketing researchers to verify existing theory and generate new insight. The findings from this study are a useful starting point to identify significant concepts and variables for further investigation.

\section{Notes}

1. Coding results are available upon request to the corresponding author.

2. A long-running American reality show that follows the Kardashian-Jenner family.

3. Emojis displayed on Twitter.

4. Username on TikTok. 


\section{References}

Frederick, E.L., \& Clavio, G. (2015). Blurred lines: An examination of high school football recruits' self-presentation on Twitter. International Journal of Sport Communication, 8(3), 330-344. doi:10.1123/IJSC.2014-0089

Geurin, A.N. (2017). Elite female athletes' perceptions of new media use relating to their careers: A qualitative analysis. Journal of Sport Management, 31(4), 345-359. doi:10. 1123/jsm.2016-0157

Geurin-Eagleman, A.N., \& Burch, L.M. (2016). Communicating via photographs: A gendered analysis of Olympic athletes' visual self-presentation on Instagram. Sport Management Review, 19(2), 133-145. doi:10.1016/j.smr.2015.03.002

Ilicic, J., \& Webster, C.M. (2016). Being true to oneself: Investigating celebrity brand authenticity. Psychology \& Marketing, 33(6), 410-420. doi:10.1002/mar.20887

Khan, G.F., \& Vong, S. (2014). Virality over YouTube: An empirical analysis. Internet Research, 24(5), 629-647. doi:10.1108/IntR-05-2013-0085

Kozinets, R. (2010). Netnography: Doing ethnographic research online. London, UK: SAGE.

Kunkel, T., \& Biscaia, R. (2020). Sport brands: Brand relationships and consumer behavior. Sport Marketing Quarterly, 29(1), 3-17. doi:10.32731/SMQ.291.032020.01

Kunkel, T., Biscaia, R., Arai, A., \& Agyemang, K. (2019). The role of self-brand connection on the relationship between athlete brand image and fan outcomes. Journal of Sport Management, 34(3), 201-216. doi:10.1123/jsm.2019-0222

Leskin, P. (2020, April 30). TikTok surpasses 2 billion downloads and sets a record for app installs in a single quarter. Business Insider. Retrieved from https://www. businessinsider.com/tiktok-app-2-billion-downloads-record-setting-q1-sensor-tower2020-4

Moran, E. (2019, December 5). How TikTok is helping sports leagues and teams better engage with female fans. Front Office Sports. Retrieved from https://frntofficesport. com/tiktok-women-sports/

Pegoraro, A. (2010). Look who's talking-Athletes on Twitter: A case study. International Journal of Sport Communication, 3(4), 501-514. doi:10.1123/ijsc.3.4.501

Pegoraro, A., \& Jinnah, N. (2012). Tweet 'em and reap 'em: The impact of professional athletes' use of Twitter on current and potential sponsorship opportunities. Journal of Brand Strategy, 1(1), 85-97.

Su, Y., Baker, B., Doyle, J., \& Kunkel, T. (2020). Rise of an athlete brand: Factors influencing the social media following of athletes. Sport Marketing Quarterly, 29(1), 33-46. doi:10.32731/SMQ.291.302020.03

Thomson, M. (2006). Human brands: Investigating antecedents to consumers' strong attachments to celebrities. Journal of Marketing, 70(3), 104-119. doi:10.1509/jmkg. 70.3 .104

Weiss, G. (2020, April 28). TikTok added 12 million unique U.S. visitors in March, as watch-time surges in quarantine. Tubefilter. Retrieved from https://www.tubefilter. com/2020/04/28/tiktok-added-12-million-unique-us-visitors-in-march/

Women's National Basketball Association. (2020). Get you a baller that can do both \#dontrushchallenge [TikTok]. Retrieved from https://www.tiktok.com/@wnba/video/ 6812727474418470149 